data-analysis software for Newark that will help to make decisions on the basis of citizen complaints. Residents tweet about the presence of potholes or broken streetlights, and an algorithm collates and summarizes the information, and suggests the most efficient way to deal with the problem. For instance, it might provide an optimized route that allows a road crew to fix the largest number of potholes in the shortest possible time, rather than sending trucks out randomly when complaints come in. Algorithms that suggest routing strategies for supplies and emergency services could also be useful after a hurricane or earthquake, and have the potential to save a lot of lives.

Newark - a densely populated city with a poverty rate of almost $30 \%$ - has a large number of vacant plots, which do not generate property tax and sometimes attract crime. Vaidya says that predictive analytics can make computer models of different actions or incentives that might entice businesses to take over those plots. Officials can also use predictive analytics to work out the best way to bring in revenue, "which in turn lets you lower the tax burden", he says.

Applying predictive analytics to government data could help police departments to work out how best to allocate their resources. It might also help to detect terrorist plots or other security threats. But relying on artificial intelligence to make official decisions without critical oversight can cause problems, Vaidya warns. For instance, a machine-

"The problem
is more
complicated
thanjust
keeping
the records
internal."
learning algorithm might build a model that relies on characteristics that are subject to antidiscrimination laws such as race or age, causing unfairness. Or it might use less-obvious proxies for race such as postcodes or the presence of certain businesses in an area.

"Everyone now is crazy about deep learning," Vaidya says, a technique that runs through many layers of mathematical calculations to mimic the way that the brain works. Among other accomplishments, it has allowed digital assistants such as Apple's Siri and Amazon's Alexa to perform reliable voice recognition, but it is not obvious even to the computer programmers how it comes up with a particular answer. "The big problem with deep learning is that you don't have an explainable model, so you basically can't understand why the algorithm is making particular choices," he adds.

\section{TEMPTING SITES}

One problem with putting information about taxes, employment and health-care access into one easy-to-access site is that the site becomes a target for hackers. "It essentially creates a honeypot," says Andrew Greenway, a partner at Public Digital, a UK consultancy that helps governments to design digital systems. "You know if you hack into that there's an enormous prize."

In 2017, e-government champion Estonia had to issue software updates for hundreds of thousands of ID cards after security researchers discovered a flaw in the cards' chip that left it vulnerable to hackers. The country's combined police and border force, which is responsible for issuing identity documents, released a statement at the time saying that they did not expect this to be the last security risk that the system will face.

Greenway, who was part of the team that set up www.gov.uk, says that government systems should segregate information, so that a hacker will have difficulty linking one piece of data about someone to another. But even if government databases remove names to obscure identity, comparing those databases to marketing lists or a telephone directory could allow some of those names to be recovered, says Eugene Spafford, a computer scientist at Purdue University in West Lafayette, Indiana. Additionally, a main component of digital government is providing citizens with easy access to their own information. "That's kind of the opposite, in a way, of trying to maintain security and privacy," Spafford says. "So the problem is more complicated than just keeping the records internal."

Computer scientists are now developing cryptographic techniques to keep data secure. One approach, which is growing in popularity because it enables data to be accessed but still protects identity, is called differential privacy. This allows a person to query a collection of records, but not to tell the difference between individual records. For example, someone could find out how many people claim a particular benefit, but not which people they are. Essentially, the computer calculates an answer, and then adds some statistical noise to obscure the source. However, it only works when numerous records are aggregated together, and it reduces the accuracy of the result. "It doesn't work everywhere, but it is extremely useful," Vaidya says. The US Census Bureau plans to use differential privacy to protect the data it collects in the 2020 Census.

\section{THE OLD WAYS}

Some of the problems with implementing digital government have little to do with technological developments. Instead, work is hampered by cost, outdated equipment, entrenched bureaucracy and a lack of political will. For instance, many digital systems still rely on archaic computer languages such as COBOL, which was created in 1959. "Some of the databases that a lot of tax systems are based on around the world are crumbling bits of COBOL that have been there for 40 years," says Greenway. "They're sort of stuck together with sticking plasters, but now they represent critical national infrastructure."

According to Spafford, some US agencies are using computers that are up to 30 years old. In

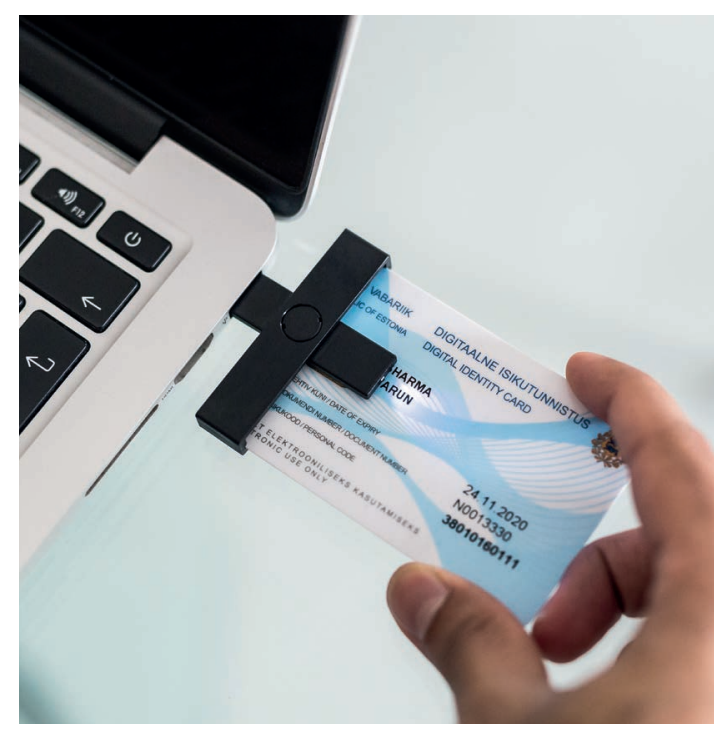

Estonia's ID cards contain chips that identify the user online - but that can pose a security risk.

2015, the US Office of Personnel Management suffered data breaches that revealed the records of 4.2 million federal employees, as well as the social security numbers of 21.5 million people who had undergone background checks for security reasons. The breach showed that the office's systems used out-of-date hardware and software with vulnerabilities that current equipment would not have. "The cost to fix it was in the many millions of dollars," he says.

Government employees are often reluctant to change the way that they operate - sometimes because laws and regulations spell out requirements for how to handle information, but also simply because of a lack of political will to change. "If they have the information, they have the power that provides," SandovalAlmazán says. "Many senior public managers don't want to lose their power, and they see the technology like a threat." Corruption can also be a problem in some places, he points out. A well-functioning e-government is not compatible with a system that thrives on bribery and personal connections.

Implementing systems is not simply a case of adapting the technology to fit the work of government. It also entails transforming government to better fit the technological nature of the twenty-first century. Government departments need to be less compartmentalized and more willing to adapt, Greenway says, and should focus more on what citizens need. In that way, e-government is not just about creating a better customer-service experience for encounters with the state. "It's about reforming the bureaucracy and the institutions, and changing some quite fundamental behaviour in the civil services," he says. Digital technology has the potential to transform the way citizens deal with government, but it could also alter the way government sees itself..

Neil Savage is a freelance writer in Lowell, Massachusetts. 